\title{
Neoliberalismus und Konstitutionalismus im Weltwirtschaftsrecht: Entstehung, Krisen, Alternativen
}

Die Regime des internationalen Wirtschaftsrechts und ihre programmatische Ausrichtung auf freie Märkte, Wettbewerb und den Abbau staatlicher Interventionen sind nicht erst seit der jüngsten Wirtschafts- und Finanzkrise in die öffentliche Kritik geraten. Vielmehr werden sie bereits seit langem von zivilgesellschaftlichen Akteuren und Entwicklungsländern wegen ihrer sozialen, ökologischen und wirtschaftlichen Auswirkungen hinterfragt. Diese Kritik ist inzwischen in der Mitte der Gesellschaft angekommen und wird auch von (ehemaligen) Vertretern dieser Regime geteilt. ${ }^{1}$

Auf semantischer Ebene wird die Kritik am Neoliberalismus von den betroffenen Organisationen aufgegriffen: So hat WTO-Generaldirektor Pascal Lamy bereits 2006 versucht, dem ausschließlich neoliberal ausgerichteten „Washington Consensus“ des Internationalen Währungsfonds (IWF) und der Weltbank einen stärker auf Ausgleich bedachten „Geneva Consensus“ des Welthandelssystems entgegen zu setzen. ${ }^{2}$ Die rechtsförmige Verarbeitung von Interessenkonflikten im Wege der Streitschlichtung eröffnet dem internationalen Handels- und Investitionsschutzrecht scheinbar die Möglichkeit, nicht-wirtschaftliche Interessen angemessen zu berücksichtigen, da ein Mechanismus für die objektive und neutrale Abwägung und den Ausgleich von Interessen zur Verfügung steht. Dieser Eindruck darf jedoch nicht darüber hinwegtäuschen, dass eventuell vorhandene Ausgleichsmechanismen nicht systemimmanent sind, sondern Folge der Kritik an der Funktionsweise von Welthandels- und Investitionsschutzrecht und somit das Ergebnis entsprechender gegenhegemonialer Bewegungen.

Trotz möglicher Öffnungen des Weltwirtschaftsrechts für nicht-wirtschaftliche Interessen sind die Regime von IWF, Weltbank und Welthandelsorganisation weiterhin darauf ausgerichtet, staatliche Interventionen in wirtschaftliche $\mathrm{Zu}$ sammenhänge zu minimieren und offene Wettbewerbsmärkte zu realisieren. Demokratisch legitimierte und sozialstaatlich motivierte Regulierung soll zugunsten der Interessen von Unternehmen und Investoren diszipliniert werden. Die Durchsetzung dieses „disziplinierenden Neoliberalismus“3 mit rechtlichen Mitteln wirkt quasi-konstitutionell. Aufgrund seiner erschwerten Abänderbarkeit legt das Handels- und Investitionsrecht nationalen Politiken verfassungsgleiche Schranken auf, die dem Schutz individueller (Wirtschafts-)Interessen dienen. ${ }^{4}$ Entstehung und Wirkungsweise, aber auch Widersprüche und Krisen des neoliberal programmierten und quasi-konstitutionell wirkenden Weltwirtschafts-

1 Stiglitz, Die Schatten der Globalisierung, 2002.

2 Lamy, Humanising Globalization, Rede am 30.1.2006 in Santiago de Chile, im Internet unter http:// www.wto.org/english/news_e/sppl_e/sppl16_e.htm (1.9.2010).

3 Gill, Power and Resistance in the New World Order, $2^{\text {nd }}$ ed., 2008, S. $137 \mathrm{ff}$.

4 Aus neoliberaler Sicht wird das begrüßt: Siehe Petersmann, Constitutional Functions and Constitutional Problems of International Economic Law, 1991. Kritisch dazu Krajewski, Verfassungsperspektiven und Legitimation des Rechts der Welthandelsorganisation, 2001. 


\section{Entstehung und Wirkungsweise neoliberalen Weltwirtschaftsrechts}

Bei der folgenden Rekonstruktion der Entstehung und Wirkungsweise des Dienstleistungshandels- und des Investitionsschutzregimes stehen intellektuelle und ideologische Bedingungen und das Interesse einzelner Akteure und Akteursgruppen im Vordergrund. Damit soll der Frage nachgegangen werden, unter welchen Bedingungen sich der disziplinierende Neoliberalismus als hegemoniale Doktrin etablieren konnte.

\section{Handel mit Dienstleistungen (GATS)}

Die Entstehung des WTO-Abkommens für den Dienstleistungshandel (General Agreement on Trade in Services, GATS) wird im Allgemeinen dadurch erklärt, dass die USA das Thema Dienstleistungshandel in den Verhandlungen der Uruguay-Runde gegen den Widerstand der Entwicklungsländer durchsetzten, weil sie sich angesichts der wachsenden Konkurrenz bei Warenproduktion und -handel durch die Schwellenländer die Realisierung komparativer Vorteile im Dienstleistungssektor versprachen. ${ }^{5}$ Konkret verlangten große US-Unternehmen der Finanz- und Telekommunikationsbranche Zugang zu den Märkten in Lateinamerika und Asien. Diese Erklärung sagt allerdings wenig über die politischen und intellektuellen Anstrengungen aus, die erforderlich waren, um Dienstleistungen überhaupt als handelsrelevantes Thema zu etablieren.

Anders als Waren sind Dienstleistungen interpersonale Austauschbeziehungen, die traditionell als nicht handelbar, da nicht lager- und transportfähig, angesehen wurden. Soweit die entsprechenden Austauschbeziehungen grenzüberschreitend stattfanden, wurden sie als ausländische Direktinvestitionen oder internationale Arbeitsverhältnisse angesehen und unterfielen nicht dem internationalen Handelssystem. Mit anderen Worten: Dienstleistungshandel musste erst erfunden werden. William Drake und Kalypso Nicolaidis haben die ideelle und politische Konstruktion des Dienstleistungshandels in den 1970er und 1980er nachgezeichnet und gezeigt, dass diese Konstruktion das Werk einer epistemischen Gemeinschaft aus einigen einflussreichen Personen aus dem Umfeld der US-amerikanischen Regierung, transnationaler Unternehmen, internationaler Organisationen wie der OECD und einzelner think tanks war. ${ }^{6}$ Durch die Gründung von Arbeitsgruppen und die Veröffentlichungen von einschlägigen Studien ${ }^{7}$ wurde so der intellektuelle Boden für ein neues Verständnis von Handel und Dienstleistungen und damit für eine „neue Realität“ gelegt. ${ }^{8}$ Erst nach der Neudefinition sozialer und wirtschaftlicher Austauschbeziehungen als handelbare Dienstleistungen konnten diese zum Regelungs- und Verhandlungsgegenstand für das multilaterale Handelssystem werden. Auf diese Weise wurden traditionelle nationalstaatliche Steuerungs- und Regulierungsmechanismen der Dienstleistungs-

5 Trebilcock/Howse, The Regulation of International Trade, $3^{\text {rd }}$ ed., 2008, S. $380 \mathrm{ff}$.

6 Drake/Nicolaidis, Ideas, Interests, and Institutionalization: „Trade in Services“ and the Uruguay Round, International Organization 1992, S. 41-69.

7 Besonders einflussreich: Feketekuty, International Trade in Services: An Overview and Blueprint for Negotiations, 1988.

8 Drake/Nicolaidis(Fn. 6), S. 45. 
produktion als „Handelsschranken“ rekonstruiert, ${ }^{9}$ auf die die Liberalisierungsund Marktöffnungsmaximen des Handelsregimes angewandt werden konnten. Staatliche und soziale Regulierung der Erbringung von Dienstleistungen wurde so einer neoliberalen Disziplinierung durch internationales Wirtschaftsrecht unterworfen. ${ }^{10}$

Die Ideengeschichte des Dienstleistungshandels ist insofern ein Beispiel dafür, dass die Etablierung neoliberaler Hegemonie nicht auf scheinbar natürlichen Gesetzmäßigkeiten beruht, sondern das Ergebnis bewusster und gewollter intellektueller und politischer Strategien ist. Es gehört zu den bemerkenswerten Phänomenen des gegenwärtigen Handelsregimes und seiner wissenschaftlichen Begleitung, dass diese Entstehungsbedingungen des GATS nahezu vergessen sind und die durch das GATS vorgenommene Definition des Dienstleistungshandels von der herrschenden Lehre und Praxis als „kanonisch“ betrachtet wird. ${ }^{11}$

Da die Konstruktion des Dienstleistungshandels nahezu ausschließlich im angloamerikanischen Raum erfolgte, waren Misstrauen und Ablehnung auf Seiten der Entwicklungsländer vorprogrammiert. Diese sahen in der neuen Thematik den Versuch der OECD-Staaten, die Agenda des Welthandelssystems zu Lasten der Entwicklungsländer auszudehnen, und konnten zunächst keine eigenen strategischen Interessen am Dienstleistungshandel erkennen. Obwohl sich diese Wahrnehmung im Laufe der Jahre änderte, führte der Widerstand gegen die Einbeziehung des Dienstleistungshandels in das Handelsregime zu einem wichtigen Konstruktionselement des GATS: Die Marktöffnungsverpflichtung und der Grundsatz der Inländerbehandlung, die über das größte Liberalisierungspotential aller GATS-Prinzipien verfügen, finden nämlich nur auf diejenigen Dienstleistungssektoren und nur $\mathrm{zu}$ denjenigen Bedingungen Anwendung, die die WTO-Mitglieder konkret festgelegt haben. ${ }^{12}$ Die WTO-Mitglieder können somit ganze Dienstleistungssektoren und einzelne Regulierungsmechanismen von den GATS-Disziplinen ausnehmen.

Dem GATS wird aufgrund dieses Mechanismus eine hohe Flexibilität und Sensibilität gegenüber der regulatorischen Autonomie der Mitglieder attestiert. ${ }^{13}$ Von dieser Flexibilität können die Mitglieder jedoch nur in laufenden Verhandlungen Gebrauch machen: Einmal zugestandene Marktöffnungen können kaum verändert oder zurückgenommen werden. Zudem geraten die von der Dienstleistungsliberalisierung ausgenommenen Bereiche und Maßnahmen in künftigen Verhandlungsrunden unter Rechtfertigungsdruck, da die WTO-Mitglieder sich verpflichtet haben, in weiteren Verhandlungsrunden die entsprechenden Einschränkungen abzubauen (Art. XIX GATS).

Auch wenn die WTO-Mitglieder Einschränkungen ihrer Liberalisierungsverpflichtungen vornehmen können, verfügen sie nicht über die Auslegungshoheit dieser Einschränkungen. In zwei wichtigen Entscheidungen hat der Appellate Body der WTO klar gestellt, dass die spezifischen Zugeständnisse integraler Bestandteil des GATS sind und dass es für deren Interpretation nicht auf die Vor-

9 Krajewski, Services Trade Liberalisation and Regulation: New Developments and Old Problems, European Yearbook of International Economic Law, 2010, 161.

10 Kelsey, Serving Whose Interests? The Political Economy of Trade in Services Agreements, $2^{\text {nd }}$ ed., 2008, S. $3 \mathrm{ff}$.

11 So pointiert Lang, GATS, in: Bethlehem u.a. (eds.), The Oxford Handbook on International Trade Law, 2009, S. 161.

12 Pitschas, Die Liberalisierung des internationalen Dienstleistungshandels im Rahmen des GATS, RIW 2003, 676.

13 Adlung, Services Negotiations in the Doha Round: Lost in Flexiblity?, Journal of International Economic Law 2006, $867 \mathrm{ff}$. 
stellungen des jeweiligen WTO-Mitglieds ankommt. ${ }^{14}$ In beiden Fällen hat der Appellate Body den Inhalt der Zugeständnisse liberalisierungsfreundlich ausgelegt und damit die Regulierungsautonomie der Staaten grundsätzlich eingeschränkt. Die Berufung der betroffenen Staaten auf spezielle Ausnahmen und Rechtfertigungstatbestände wies er dabei überwiegend zurück. Es zeigt sich somit, dass Reichweite und Umfang der disziplinierenden Wirkung des WTORechts im Einzelfall von den WTO-Streitschlichtungsinstitutionen bestimmt werden, da die vorhandenen Ausgleichsmechanismen im WTO-Recht von diesen Gremien interpretiert und angewendet werden.

\section{Schutz ausländischer Investitionen}

Das internationale Rechtsregime zum Schutz ausländischer Investitionen besteht aus gewohnheitsrechtlichen Grundsätzen sowie bilateralen, regionalen und multilateralen Verträgen. Seine disziplinierende Wirkung gegenüber staatlicher Regulierung entfaltet das Regime vor allem durch sog. bilaterale Investitionsschutzverträge (Bilateral Investment Treaties, BITs). Deren Zahl ist von knapp 400 in den 1980er Jahren auf rund 2700 im Jahre 2009 angewachsen..$^{15}$ Deutschland gehört mit aktuell (Stand: August 2010) 130 derartigen Abkommen zu den Staaten mit den meisten BITs. Neben die BITs treten einige regionale Verträge mit Investitionsschutzklauseln wie das NAFTA-Abkommen oder der EnergiechartaVertrag, auf dessen Grundlage das Energieunternehmen Vattenfall jüngst gegen umweltschutzrechtliche Auflagen für den Bau eines neuen Kohlekraftwerks in Hamburg vorgehen wollte. ${ }^{16}$

Trotz der dem Grunde nach bilateralen Investitionsschutzbeziehungen, die durch ein BIT begründet werden, ist das internationale Investitionsschutzrecht de facto ein multilaterales Regime, da nahezu alle Verträge ähnliche Grundprinzipien enthalten, die in der Praxis weitgehend ähnlich interpretiert werden. ${ }^{17} \mathrm{In}-$ haltlich enthalten BITs Verpflichtungen zum Schutz der Interessen und Rechtsgüter ausländischer Investoren. Diese reichen von Ersatzansprüchen bei Enteignungen über Grundsätze der fairen Behandlung und Nichtdiskriminierungsprinzipien bis zum freien Kapitaltransfer im Zusammenhang mit Investitionen. ${ }^{18}$ Anders als das Welthandelsrecht verfügen diese Abkommen typischerweise über keine ausdrücklichen Ausnahme- oder Rechtfertigungstatbestände, die eine Abwägung unterschiedlicher Interessen vorsehen oder ermöglichen. Soweit derartige Abwägungen gleichwohl erfolgen (sollen), ist eine entsprechende Interpretation der Tatbestandsbegriffe („Maßnahme, die einer Enteignung gleichkommt“; „gerechte und billige Behandlung“, „,weniger günstige Behandlung") erforderlich.

Ihre besondere disziplinierende Funktion entfalten Investitionsschutzabkommen durch ihre innovative Form der Streitbeilegung. Während die Abkommen noch bis in die 1980er Jahre typischerweise nur klassische zwischenstaatliche Streitbeilegungsverfahren vorsahen, enthalten inzwischen die meisten Abkommen die Möglichkeit sog. Staat-Investor-Schiedsverfahren. Mit diesen kann ein

14 United States - Measures Affecting the Cross-Border Supply of Gambling and Betting Services, WT/ DS285/AB/R, 20. April 2005 und China - Measures Affecting Trading Rights and Distribution Services for Certain Publications and Audiovisual Entertainment Products, WT/DS363/AB/R, 21. Dezember 2009.

15 UNCTAD, IIA Monitor No. 3 (2009): Recent developments in international investment agreements (2008-June 2009), 2009, 2.

16 Vattenfall/Germany, ICSID Case No. ARB/09/6. Der Streitfall wurde Ende August 2010 gütlich beigelegt; Details der Einigung sind bislang nicht bekannt.

17 Schill, The Multilateralization of International Investment Law, 2009.

18 Überblick bei Griebel, Internationales Investitionsrecht, 2008, S. 62 ff. 
ausländischer Investor Maßnahmen des Gaststaates von einem internationalen Schiedsgericht auf ihre Vereinbarkeit mit dem Abkommen überprüfen zu lassen. Regelmäßig wird dabei auf die Schiedsregeln des bei der Weltbank angesiedelten Internationalen Zentrums zur Beilegung von Investitionsstreitigkeiten ICSID verwiesen. ${ }^{19}$ Ein ausländischer Investor erhält dadurch die im Völkerrecht nahezu einmalige Möglichkeit, unter Verzicht auf lokale Rechtsmittel gegen staatliche Maßnahmen direkt vor einem internationalen Schiedsgericht vorzugehen. Die Zahl derartiger Verfahren hat in den letzten Jahren sprunghaft zugenommen; inzwischen sind mehr als 300 Streitigkeiten anhängig. ${ }^{20}$ Die besondere Attraktivität von Investor-Staat-Schiedsverfahren beruht auf der verhältnismäßig kurzen Verfahrensdauer, der direkten Vollstreckbarkeit der Titel und der Möglichkeit, nach dem Grundsatz „Dulde und liquidiere“ für jede Art von Rechtsverletzung Schadensersatz zu verlangen.

Die Verfahrensregeln der Investitionsschiedsgerichtsbarkeit sind der Handelsschiedsgerichtsbarkeit nachgebildet. Die Beratungen sind nicht öffentlich und die Entscheidungen grundsätzlich keiner weiteren gerichtlichen Überprüfung unterworfen. Die Zusammensetzung der Schiedsgerichte wird durch die Parteien bestimmt; eine öffentliche Kontrolle findet kaum statt. ${ }^{21}$ Eine Vielzahl der Schiedsrichter stammt aus der unternehmensnahen anwaltlichen Praxis und ist auch in der kommerziellen Schiedsgerichtsbarkeit tätig. Der Kreis der Schiedsrichter ist klein, da die persönliche Reputation von großer Bedeutung ist. Die Mitglieder dieser epistemischen Gemeinschaft sind durch ähnliche Ausbildungen, berufliche Sozialisationen und Wertvorstellungen geprägt. Die Auslegung des Investitionsrechts durch die Schiedsgerichte ist daher grundsätzlich investorfreundlich. Auf diese Weise wird das Recht auf der Grundlage eines bestimmten Vorverständnisses im Sinne der privaten Interessen der Investoren reproduziert.

Die Investitionsschiedsgerichtsbarkeit hat so eine neue konstitutionelle Dimension $^{22}$ des internationalen Wirtschaftsrechts geschaffen, deren disziplinierende Wirkung bei Verfahren gegen Sozialregulierung wie Gesundheits- und Umweltschutzmaßnahmen oder die Bewältigung der Folgen von Privatisierungen besonders deutlich wird. ${ }^{23}$ Die Grundfrage des Investitionsschutzrechts lautet nämlich stets, ob derartige Maßnahmen unverhältnismäßig stark die wirtschaftlichen Interessen von Investoren beeinträchtigen. Private Eigentumsrechte werden somit gegen öffentliche Politiken in Stellung gebracht und mit Hilfe der Schiedsgerichte durchgesetzt.

\section{Krisen und Widersprüche}

Trotz der soeben skizzierten effektiven Durchsetzung des disziplinierenden Neoliberalismus im GATS- und Investitionsschutzrecht sind beide Regime nicht krisenfest und werden durch innere Widersprüche gekennzeichnet.

19 Dazu Schöbener/Markert, Das International Centre for Settlement of Investment Disputes (ICSID), ZVglRWiss 2006, 65-116.

20 UNCTAD, Latest Developments in Investor-State Dispute Settlement, IIA Issues Note No. 1, 2010, 2.

21 Zum Ganzen Van Harten, Investment Treaty Arbitration and Public Law, 2007.

22 Ausführlich dazu Schneiderman, Constitutionalizing Economic Globalization - Investment Rules and Democracy's Promise, 2008, S. $37 \mathrm{ff} ., 74 \mathrm{ff}$.

23 Vgl. z.B. Metalclad/Mexico, ICSID Case No. ARB(AF)/97/1; Tecmed/Mexico, ICSID Case No. ARB(AF)/00/2; Azurix/Argentina, ICSID Case No. ARB/01/12; Biwater Gauff/Tanzania, ICSID Case No. ARB/05/22. 
Zwar erweisen sich Welthandels- und Investitionsschutzregime gegenüber der Infragestellung neoliberaler Ideologien in Folge der jüngsten Wirtschafts- und Finanzkrise (noch) als bemerkenswert resistent. In der Welthandelsorganisation (WTO) sorgt man sich hauptsächlich über die Gefahr eines neuen Protektionismus und handelsdiskriminierende oder wettbewerbsverzerrende Wirkungen von wirtschaftlichen Unterstützungsprogrammen. Das Paradigma der Handelsliberalisierung wird nicht in Frage gestellt. ${ }^{24}$ Die von der Stiglitz-Kommission der Vereinten Nationen im September 2009 formulierte Kritik an der Liberalisierungsphilosophie des Welthandels- und Investitionsrechts ${ }^{25}$ hat sowohl in den jeweiligen Institutionen als auch in der wissenschaftlichen Debatte noch kaum Gehör und erst recht noch keine Entsprechung gefunden.

Dieses Festhalten am Paradigma der Handelsliberalisierung kann jedoch nicht darüber hinwegtäuschen, dass sich die WTO und das multilaterale Handelssystem seit gut einem Jahrzehnt in einer Dauerkrise befinden, die durch nichts besser charakterisiert wird als durch die wiederholten und immer wieder gescheiterten Versuche, neue Liberalisierungsverhandlungen erfolgreich zu einem Abschluss zu bringen. Bereits der ursprünglich für 1999 geplante Auftakt der Verhandlungen musste um zwei Jahre auf 2001 verschoben werden. Nach und nach wurden die Verhandlungen von „neuen Themen“ wie Wettbewerbs- und Investitionsschutz befreit. Auch nach dem vorübergehenden Aussetzen der Verhandlungen im Juni 2006 und ihrer Wiederaufnahme Anfang 2007 sind die WTO-Mitglieder von einer Einigung weit entfernt. Dies gilt auch für die Verhandlungen zur weiteren Liberalisierung des Dienstleistungshandels, die sich seit gut fünf Jahren kaum bewegt haben. Beobachter gehen inzwischen davon aus, dass selbst bei einem Abschluss der Verhandlungen kein substantieller Liberalisierungsschub $\mathrm{zu}$ erwarten ist. ${ }^{26}$

Ansätze in der Literatur, diese Krise des GATS jenseits der altbekannten Vorwürfe gegen angeblich zu protektionistische Regierungen (und neuerdings auch gegen NGOs) zu erklären, finden sich selten. Stattdessen wird gebetsmühlenartig für weitere Dienstleistungsliberalisierungen geworben. ${ }^{27}$ Dadurch bleibt der Blick auf die inneren Widersprüche des GATS und der Liberalisierung des Dienstleitungshandels verstellt. Untersucht man die Entwicklung des GATS in seinem zeitgeschichtlichen Kontext näher, erkennt man, dass die Krise des GATS der Krise des Privatisierungs- und Liberalisierungsdogmas auf nationalstaatlicher Ebene entspricht. Hier schließt sich der Kreis: Die Krise des Neoliberalismus und seiner Maximen ist längst in der WTO angekommen und beeinträchtigt ihre Funktionsweise weitaus mehr als die Aufarbeitung der Auswirkungen der Wirtschafts- und Finanzkrise.

Trotz seiner beispiellosen materiellen und prozessualen Expansion ist auch das Investitionsschutzregime nicht von Krisen verschont geblieben. Erstmals offenbar wurde dies im Kontext der gescheiterten Verhandlungen über ein multilaterales Investitionsschutzabkommen (MAI) in der OECD. ${ }^{28}$ In jüngster Zeit hat vor allem die Einschränkung nationaler Regelungsautonomie durch schiedsge-

24 WTO, Trade Policy Review Body, Report to the TPRB from the Director-General on Trade-related Developments, 14. Juni 2010, WT/TPR/OV/W/3.

25 United Nations, Report of the Commission of Experts of the President of the United Nations General Assembly on Reforms of the International Monetary and Financial System, September 2009, Chapter 3, Absatz 208.

26 Jara/Dominguez, Liberalization of Trade in Services and Trade Negotiations, Journal of World Trade 2006, 113.

27 Mattoo, Services in a Development Round: Three Goals and Three Proposals, Journal of World Trade, 2005, 1223 ff.; Leal-Arcas, Services as Key for the Conclusion of the Doha Round, Legal Issues of Economic Integration 2008, $301 \mathrm{ff}$.

28 Dazu Karl, Das Multilaterale Investitionsabkommen (MAI), RIW 1998, 432-440. 
richtliche Entscheidungen zu einer gegenüber der bisherigen Ausrichtung des Systems kritischen Gegenbewegung geführt. ${ }^{29}$ Diese wurde anlässlich der Kündigung des ICSID-Übereinkommens durch Bolivien im Mai 2007 und durch Ecuador im Juli 2009 besonders sichtbar. ${ }^{30}$

Welche Neuausrichtungen sind angesichts dieser Krisen möglich und angebracht? In Praxis und Literatur am weitesten verbreitet ist die Auffassung, dass es sich bei der WTO-Krise um eine Krise des Multilateralismus (und nicht des Neoliberalismus) handele. Das wirft die Frage auf, ob regionale Integrationsbestrebungen Alternativen zum gegenwärtigen System sein können.

\section{Regionalisierung als Alternative?}

Seit dem Inkrafttreten der WTO-Abkommen ist eine bemerkenswerte Proliferation regionaler und bilateraler Handelsabkommen $\mathrm{zu}$ beobachten. ${ }^{31}$ Im Juli 2010 waren 193 derartiger Abkommen in Kraft, darunter regionale Integrationsabkommen wie NAFTA, Mercosur oder die Südafrikanische Entwicklungsgemeinschaft SADC, sowie zahlreiche bilaterale Abkommen wie z. B. das Assoziierungsabkommen zwischen der EU und Chile von 2002. Ob diese Abkommen eine Alternative zum neoliberalen Modell der WTO und des Investitionsschutzrechts darstellen, hängt in erster Linie von ihrer programmatischen Ausrichtung ab. Um diese ermitteln zu können, bedarf es einer Analyse der jeweiligen Inhalte der Abkommen.

Hierbei macht sich mit Blick auf regionale Abkommen, die Vorschriften zum Dienstleistungshandel enthalten, schnell Ernüchterung breit. Die meisten Abkommen sind sog. "GATS Plus"-Abkommen, d. h. sie enthalten weitergehende Liberalisierungsverpflichtungen als das GATS selbst. Sie beruhen auf der gleichen Konzeption des Dienstleistungshandels wie das GATS und enthalten vergleichbare Verpflichtungen zu Marktöffnung und Nichtdiskriminierung sowie zum Abbau handelshemmender Regulierungen. Dies gilt sowohl für Abkommen zwischen Industrie- und Entwicklungsländern als auch für Abkommen von Entwicklungsländern untereinander wie das Rahmenabkommen über Dienstleistungshandel der ASEAN-Staatengemeinschaft von 1995 oder das Montevideo Protocol über Dienstleistungshandel des Mercosur von 1997. Diese Ausrichtung der regionalen Abkommen ist kein Zufall, sondern wird auch dadurch bestimmt, dass die jeweiligen Vertragspartner überwiegend auch Mitglieder der WTO sind. Das GATS erlaubt regionale Handelsabkommen (ähnlich wie das GATT) jedoch nur, wenn diese insgesamt zu keinen höheren Handelsbeschränkungen führen. ${ }^{32}$ Damit enthält das WTO-Recht einen „konstitutionellen Hebel“, mit dessen Hilfe das Liberalisierungsparadigma in regionalen Handelsabkommen verankert werden kann.

Die meisten regionalen Abkommen unterscheiden sich allerdings dadurch von WTO und GATS, dass ihre Streitbeilegungsmechanismen weniger durchsetzungsstark sind. Sie gleichen oft noch traditionellen völkerrechtlichen Methoden,

Kaushal, Revisting History: How the Past Matters for the Present Backlash Against the Foreign Investment Regime, Harv Int L J 2009, $492 \mathrm{ff}$.

30 Nowrot, International Investment Law and the Republic of Ecuador: From Abitral Bilateralism to Judicial Regionalism, in: Tietje/Kraft/Lebmann (Hrsg.), Beiträge zum Transnationalen Wirtschaftsrecht, 2010, S. $5 \mathrm{ff}$.

31 Fiorentino/Verdeja/Toqueboeuf, The Changing Landscape of Regional Trade Agreements: 2006 update, WTO Discussion Paper No. 12, 2007.

32 S. dazu Cottier/Molinuevo, Article V GATS, in: Wolfrum/Stoll/Feinängle (Hrsg.), WTO - Trade in Services, 2008, S. $125 \mathrm{ff}$. 
die den Konsens der Streitparteien erfordern und nicht wie das WTO-Verfahren auch gegen den Willen (einer) der Parteien durchgesetzt werden kann. ${ }^{33}$ Die rechtsförmige Durchsetzung des disziplinierenden Neoliberalismus ist daher in diesen Zusammenhängen schwächer ausgeprägt. Hierin kann man jedoch nur schwer einen alternativen oder gar emanzipatorischen Ansatz gegenüber dem multilateralen System sehen. Der Rückgriff auf eher traditionelle Streitbeilegungsmethoden in regionalen Handelsabkommen dürfte vor allem damit zusammenhängen, dass die Abkommen keine institutionellen Strukturen vorsehen, die ein der WTO vergleichbares Streitbeilegungssystem verwalten könnten. Im Ergebnis lässt sich daher festhalten, dass in den gegenwärtigen regionalen Handelsabkommen keine Ansätze für Alternativen zur neoliberalen Hegemonie des Welthandelssystems zu finden sind.

Dagegen könnten jüngste investitionspolitische Entwicklungen in Lateinamerika in eine andere Richtung führen. Nicht erst seit dem Rückzug Ecuadors und Boliviens aus dem ICSID-System wird dort verstärkt über regionale Alternativen zum herrschenden System nachgedacht. ${ }^{34}$ Insbesondere die Bestrebungen der Bolivarianischen Allianz für die Völker unseres Amerika (ALBA) verdienen in diesem Zusammenhang Beachtung. ${ }^{35}$ Dieser Zusammenschluss von neun Staaten Südamerikas und der Karibik ${ }^{36}$ beabsichtigt eine neue, nicht neoliberale Form der regionalen Integration und eine größere Unabhängigkeit der Region von den USA und Europa. Der thematische Zuschnitt des Bündnisses geht weit über traditionelle Handels- und Wirtschaftsintegration hinaus und umfasst soziale, ökologische und entwicklungspolitische Ziele. Damit kann das ALBA-Projekt auch als Gegenentwurf zu auf Handelsliberalisierung und Marktintegration ausgerichteten regionalen Bündnissen wie z. B. dem MERCOSUR gesehen werden. Neben der jüngst realisierten Etablierung der Regionalwährung SUCRE als Grundlage für regionale Handelsbeziehungen plant ALBA die Errichtung eines eigenen regionalen Schiedsgerichtszentrums zur Beilegung von Investitionsstreitigkeiten als Alternative zum ICSID-System. ${ }^{37}$ Auch der elf lateinamerikanische Energieminister umfassende Energierat Südamerikas (Energy Council of South America) hat eine Arbeitsgruppe etabliert, die die Errichtung eines neuen Streitbeilegungsmechanismus für Investitionsstreitigkeiten prüfen soll. ${ }^{38}$

Den genannten Initiativen sind bislang zwar noch wenig konkrete Ergebnisse gefolgt, und die Konturen möglicher Alternativen bleiben vorerst vage. Es kann jedoch angenommen werden, dass versucht wird, das Verhältnis von Investorenrechten und staatlicher Regulierungsautonomie neu zu justieren und die einseitige Ausrichtung des bisherigen Systems zu Gunsten einer stärkeren Berücksichtigung öffentlicher Interessen und Belange zu verändern. Dies kann durch ausdrückliche Bezugnahmen auf regulatorische Ziele und Instrumente in den Abkommen oder durch eine Orientierung der Streitschlichtung an den Prinzipien einer demokratisch legitimierten, öffentlich zugänglichen Gerichtsbarkeit erfolgen. Sollten die genannten Initiativen zu praktischen Veränderungen führen, ist auch damit zu rechnen, dass sich die Schiedsrichter und Streitschlichter nicht

Fink/Molinuevo, East Asian Free Trade Agreements in Services: Key Architectural Elements, J Int Economic Law 2008, $296 \mathrm{ff}$.

34 Nowrot (Fn. 30), S. 7 f.

35 Fechner, Die Bolivarianische Allianz ALBA und die sozialen Bewegungen, KJ 2010, $59 \mathrm{ff}$.

36 Mitglieder sind Antigua und Barbuda, Bolivien, Dominica, Ecuador, Honduras, Kuba, Nicaragua, St. Vincent und die Grenadinen sowie Venezuela, dessen Präsident Chavez die Initiative zu ALBA ergriff und sie maßgeblich beeinflusste. Siehe allgemein http://www.alianzabolivariana.org/ (2.9.2010).

37 Diaz, ALBA moves forward with plan to create regional investment arbitration alternative to ICSID at 7th Summit, Investment Treaty News, November 2009.

Nowrot (Fn. 30), S. 8. 
aus der gleichen Gruppe rekrutieren werden wie das gegenwärtige Personal von Investitionsschiedsgerichten.

Die weiteren Entwicklungen werden Aufschluss darüber geben, inwieweit sich die neuen regionalen Initiativen zu einem gegenhegemonialen Projekt entwickeln können und wie sich dies auf das herrschende System des disziplinierenden Neoliberalismus auswirken wird. Schon jetzt ist jedoch deutlich, dass Regionalisierung an sich kein Gegenprojekt ist, sondern dass es auf die Inhalte und mehr noch auf die den Projekten zu Grunde liegenden Wertvorstellungen und Überzeugungen ankommt.

\section{Auf dem Weg zu einem postneoliberalen Weltwirtschaftsrecht}

Mit den letztgenannten Überlegungen klingt die abschließende These der vorliegenden Betrachtungen bereits an. Für die Herausbildung einer postneoliberalen Rechtsordnung in den internationalen Wirtschaftsbeziehungen, mit der der disziplinierende Neoliberalismus überwunden werden kann, kommt es weniger darauf an, ob und welche neuen Rechtsregeln etabliert werden. Entscheidender ist, mit welchen Vorverständnissen und Zielen das Recht interpretiert und angewandt wird. Erforderlich ist nicht unbedingt ein anderes positives Recht, sondern ein neues Verständnis und ein neuer Umgang mit dem Recht. Dazu muss das bestehende internationale Wirtschaftsrecht für ein neues postneoliberales Verständnis geöffnet werden. ${ }^{39}$

Die in diesem Beitrag skizzierten Probleme und Krisen des Weltwirtschaftsrechts und seine neoliberale Ausrichtung beruhen zu einem großen Teil auf der Interpretation des Rechts durch den herrschenden juristischen Diskurs und seiner Anwendung im Rahmen einseitig auf Handelsliberalisierung und Investitionsschutz gerichteten Politiken. Konkret: Begriffe und Phrasen wie „weniger schlechte Behandlung“, „nicht mehr handelsbeschränkend als notwendig“, „Maßnahme, die einer Enteignung gleichkommt“ usw. sind offene Rechtsbegriffe, die semantisch nicht auf eine neoliberale Programmatik festgelegt sind. Sie werden vor allem durch Interpretation und Auslegung zu normativen Vorgaben, mit denen staatliche Regulierung diszipliniert werden kann. Insofern entfaltet sich der disziplinierende Neoliberalismus im geltenden Weltwirtschaftsrecht durch die Besetzung offener Begriffe mit bestimmten neoliberalen Inhalten. Die Orientierung des internationalen Wirtschaftsrechts an diesen Inhalten konnte sich etablieren und bislang bestehen bleiben, weil sie auf einem hegemonialen Konsens der beteiligten Akteure (Diplomaten, Schiedsrichter und Wissenschaftler) beruhte, der als „herrschende Meinung“ weitgehend anerkannt und unbestritten blieb. 40

Hieraus folgt, dass der Weg zu einer postneoliberalen Rechtsordnung nicht in erster Linie eine Änderung des positiven Rechts erfordert, sondern vor allem eine Neuausrichtung seiner Auslegung und Anwendung. Diese muss durch die Ausarbeitung und Anwendung alternativer Deutungen der jeweiligen Rechtsbegriffe sowohl in juridischen Foren (Streitbeilegungsverfahren) als auch in sozialen Auseinandersetzungen erkämpft werden. Vor diesem Hintergrund ist die Etablierung eines alternativen Streitbelegungsmechanismus für Investitionsstreitig-

39 In diesem Sinne bezüglich des GATS und der Regulierung von Finanzdienstleistungen Cottier/Krajewski, Which Role for Non-Discrimination and Prudential Standards in International Financial Law?, J Int Econ Law, 2010, im Erscheinen.

40 Zu den hegemonialen Prozessen der Bildung von „herrschender Meinung “ klassisch Wesel, hM, in: ders., Aufklärungen über Recht. Zehn Beiträge zur Entmythologisierung, 1981, S. 14 ff. 
keiten in Lateinamerika ein Schritt in die richtige Richtung, da er das Interpretationsoligopol der bisherigen Schiedsgerichte aufbrechen und Raum für neue Auslegungen schaffen kann. Eine gegenhegemoniale Strategie sollte zudem mit der Kritik an den einschlägigen Urteilen und Entscheidungen der WTO und der Investitionsschiedsgerichte einhergehen und aufzeigen, dass, warum und mit welchen Folgen Rechtsbegriffe in einer bestimmten Weise interpretiert und angewendet werden. Hierzu kann kritische Rechtswissenschaft einen wichtigen Beitrag leisten.

\section{Festschrift}

für Heide Pfarr

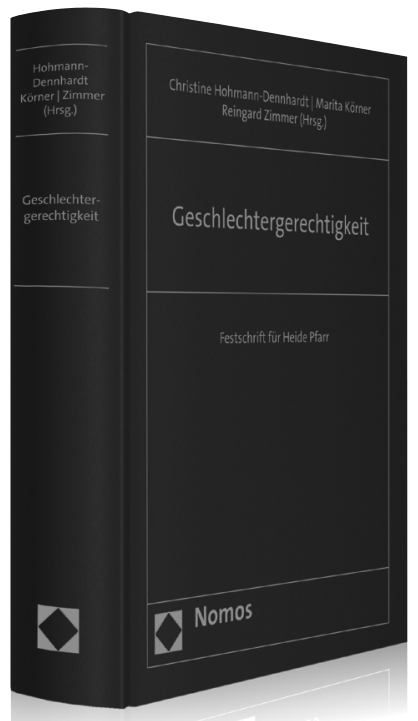

Geschlechtergerechtigkeit

Festschrift für Heide Pfarr

Herausgegeben von RiBVerfG

Dr. Christine Hohmann-Dennhardt, Prof. Dr. Marita Körner und Dr. Reingard Zimmer

2010, 545 S., geb., 109, - $€$

ISBN 978-3-8329-5854-1

Die Festschrift konzentriert sich auf eines der zentralen Forschungsfelder der Jubilarin: Geschlechtergerechtigkeit. Thematisiert wird u.a. Geschlechtergerechtigkeit auf dem Arbeitsmarkt, Antidiskriminierung und Gleichstellung sowie die Vereinbarkeit von Beruf und Familie. Der Blick über die Landesgrenzen verdeutlicht die europäische und internationale Relevanz des Themas.

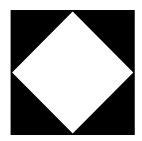

